\title{
CONTROLLABILITY OF NONLINEAR VOLTERRA INTEGRODIFFERENTIAL SYSTEMS WITH PRESCRIBED CONTROLS ${ }^{1}$
}

\author{
K. BALACHANDRAN and D. LALITHA \\ Bharathiar University \\ Department of Mathematics \\ Coimbatore 641046 \\ Tamil Nadu \\ INDIA
}

\begin{abstract}
Sufficient conditions for the global controllability of nonlinear Volterra integrodifferential systems with prescribed controls are derived. The method is a transformation of the given control system into a boundary value problem and then the result is obtained by the application of the Schaefer fixed point theorem.

Key words: Controllability, Volterra Integrodifferential Systems, Boundary Value Problem, Fixed Point Theorem.
\end{abstract}

AMS (MOS) subject classifications: $\quad 93 \mathrm{~B} 05$.

\section{INTRODUCTION}

Controllability of nonlinear systems has been studied by several authors by means of fixed point principles [4]. Anichini [1,2], Dauer [7], Kartsatos [8] and Lukes [9] respectively studied the controllability of nonlinear systems with prescribed controls by means of Schaefer's theorem, Fan's theorem, Tychonov's theorem and the Schauder theorem. With the use of Fan's fixed point theorem, Dauer [6] established sufficient conditions for the controllability of nonlinear systems between two fixed points. Chukwu and Silliman [5] examined the problem for nonlinear systems to a closed target set. Balachandran [3] studied the problem for the nonlinear Volterra integrodifferential systems.

In this paper, we seek to find sufficient conditions for the nonlinear control process,

$$
\dot{x}=A(t) x+\int_{0}^{t} H(t, s) x(s) d s+B(t) u(t)+g(t, x(t))
$$

is completely controllable in a finite interval $\left[t_{0}, T\right]$ by means of controls whose initial and final values can be assigned in advance. That is, we want to find conditions upon $A(t), B(t)$,

\footnotetext{
${ }^{1}$ Received: April, 1991. Revised: July, 1991.
} 
$H(t, s), g(t, x)$ which ensure that, for each $t_{0}, T \in \mathbb{R}, \alpha, \beta \in \mathbb{R}^{m}, x_{0}, x_{T} \in \mathbb{R}^{n}$, there exists a control $u \in C\left(\left[t_{0}, T\right] ; \mathbb{R}^{m}\right)$ of $(1)$, with $u\left(t_{0}\right)=\alpha, u(T)=\beta$ which produces a response $x(t, u)$ satisfying the boundary conditions $x\left(t_{0}, u\right)=x_{0}$ and $x(T, u)=x_{T}$.

This result will be obtained by an application of a fixed point argument to the linear boundary value problem

$$
\begin{gathered}
\dot{x}=A(t) x+\int_{0}^{t} H(t, s) x(s) d s+B(t) u(t)+g(t, z(t)) \\
x\left(t_{0}\right)=x_{0}, \quad x(T)=x_{T} \\
u\left(t_{0}\right)=\alpha, \quad u(T)=\beta
\end{gathered}
$$

where $z \in C\left(\left[t_{0}, T\right] ; \mathbb{R}^{n}\right)$, the space of continuous functions with sup norm. For brevity let us take $t_{0}=0, \alpha=u_{0}$ and $\beta=u_{T}$.

This result generalizes the results of Anichini [1].

\section{PRELIMINARIES}

Consider the nonlinear Volterra integrodifferential system

$$
\dot{x}(t)=A(t) x(t)+\int_{0}^{t} H(t, s) x(s) d s+B(t) u(t)+g(t, x(t))
$$

where the state $x$ is an $n$-vector and the control $u$ is an $m$-vector.

The matrix functions $\quad A: J \rightarrow \mathbb{R}^{n^{2}}, \quad B: J \rightarrow \mathbb{R}^{n m}, \quad J=[0, T], \quad H: \Delta \rightarrow \mathbb{R}^{n^{2}}$, $\Delta=\{(t, s): 0 \leq t \leq s \leq T\}$ are assumed to be continuous. The function $g:[0, T] \times \mathbb{R}^{n} \rightarrow \mathbb{R}^{n}$ is such that $g(t, x) \in C^{1}\left(J \times \mathbb{R}^{n}, \mathbb{R}^{n}\right)$. The norm of a matrix and a function are taken as in [1]. Assume that, for $t \in J$, and $(t, s) \in \Delta,\|A(t)\| \leq a,\|B(t)\| \leq b,\|H(t, s)\| \leq c$ and $\|g(t, x)\| \leq \gamma(t)$, where $\gamma(t)$ is a continuous function and $a, b, c$ are positive constants. We observe that the hypothesis on $A(t)$ and $H(t, s)$ allow us to say that there exists an unique continuous matrix $E(t, s)$ such that

$$
\frac{\partial E(t, s)}{\partial s}+E(t, s) A(s)+\int_{0}^{t} E(t, w) H(w, s) d w=0
$$

with $E(t, t)=I$, the identity matrix.

Following Anichini [1,2], we say that $(1)$ is $[0, T]$-controllable by means of a certain set $U$ of controls iff, for every pair $x_{0}, x_{T} \in \mathbb{R}^{n}$, there exists $u \in U$, such that $x(0, u)=x_{0}$, 
$x(T, u)=x_{T}$

For brevity let us denote

$$
\begin{gathered}
P(t ; \theta)=\int_{0}^{t} E(\theta, \theta-s) B(\theta-s) d s \\
\bar{C}(t ; T)=\int_{T-t}^{T} P(s ; T)^{*} d s-(t / T) \int_{0}^{T} P(s ; T)^{*} d s \\
S(t ; T)=\int_{0}^{t} E(t, s) B(s) \bar{C}(s ; T) d s
\end{gathered}
$$

and define

$$
\begin{gathered}
M(0, t)=\int_{0}^{t} B(s) B(s)^{*} d s \\
\bar{S}(T)=\int_{0}^{T} P(s ; \theta) P(s ; \theta)^{*} d s-(1 / T)\left[\int_{0}^{T} P(s ; \theta) d s\right]\left[\int_{0}^{T} P(s ; \theta)^{*} d s\right]
\end{gathered}
$$

where the star denotes the matrix transpose. Observe that $P(t ; \theta), \bar{C}(t ; T)$ and $S(t ; T)$ are continuous.

We use the following theorem, due to Schaefer.

Proposition 1: Let $X$ be a normed space, $T$ a continuous mapping of $X$ into $X$, which is compact on each bounded subset of $X$. Then, either $(i)$ the equation $x=\lambda T(x)$ has a solution for $\lambda=1$, or (ii) the set of all such solutions $x$ for $0<\lambda<1$, is unbounded.

The following theorem is vital to the criterion of controllability.

Theorem 1: $\quad$ Assume that the control process (1) satisfies the hypotheses. If the matrix $M\left(0, t_{1}\right)$ is non-singular for some $t_{1}>0$, then the set of points attainable by the trajectories of the control process (1) is all of $\mathbb{R}^{n}$.

Proof: $\quad$ For fixed $u$, the given system has a solution $x(t ; u)$ which satisfies

$$
\begin{aligned}
x(t ; u)=x_{0} & +\int_{0}^{t} A(s) x(s ; u) d s+\int_{0}^{t}\left[\int_{0}^{s} H(s, \tau) x(\tau ; u) d \tau\right] d s \\
& +\int_{0}^{t} B(s) u(s) d s+\int_{0}^{t} g(s, x(s ; u)) d s .
\end{aligned}
$$


Let $x_{1}$ be any given point in $\mathbb{R}^{n}$. We have to find a control $v$ such that for some finite point $t_{1}>0, x\left(t_{1} ; v\right)=x_{1}$. Consider the controls of the form $v(t)=B(t)^{*} q$, where $q \in \mathbb{R}^{n}$. Define a mapping $S: \mathbb{R}^{n} \rightarrow \mathbb{R}^{n}$ as follows:

$$
\begin{gathered}
S(q)=M^{-1}\left(0, t_{1}\right)\left[x_{1}-K(q)-x_{0}\right] \text { where } \\
K(q)=\int_{0}^{t_{1}} A(s) x(s ; q) d s+\int_{0}^{t_{1}}\left[\int_{0}^{t_{1}} H(s, \tau) x(\tau, q) d \tau\right] d s+\int_{0} g(s, x(s ; q)) d s .
\end{gathered}
$$

Suppose that the mapping $q \rightarrow S(q)$ has a fixed point. Then $q=M^{-1}\left(0, t_{1}\right)\left[x_{1}-K(q)-x_{0}\right]$ and from (3) it is very easy to verify that $x\left(t_{1}, q\right)=x_{1}$.

Now we shall prove the mapping $q \rightarrow S(q)$ has a fixed point. Since all the functions involved in the definition of the operator $S$ are continuous, this mapping is continuous. From (3) we have

$$
\begin{gathered}
\|x(t ; u)\| \leq\left\|x_{0}\right\|+\int_{0}^{t} a\|x(s ; u)\| d s+\int_{0}^{T}\left[\int_{0}^{s} c\|x(\tau ; u)\| d \tau\right] d s \\
+\int_{0}^{T} \sup \{b\|u(s)\|, s \in[0, T]\} d s+\int_{0}^{T} \gamma(s) d s \\
\leq \alpha_{0}+\int_{0}^{t}\left[a+\int_{0}^{s} c d \tau\right]\|x(\tau ; u)\| d s
\end{gathered}
$$

where

$$
\alpha_{0}=\left\|x_{0}\right\|+b \int_{0}^{T} \sup \{\|u(s)\|, s \in[0, T]\} d s+\int_{0}^{T} \gamma(s) d s
$$

Therefore by Gronwall's inequality

$$
\begin{gathered}
\|x(t ; u)\| \leq \alpha_{0} \exp \left[\int_{0}^{t}\left(a+\int_{0}^{s} c d \tau\right) d s\right] \\
\leq \alpha_{0} \exp \left(a T+c T^{2} / 2\right) .
\end{gathered}
$$

Thus if $\|q\|<+\infty$, then $\|x(t ; u)\|<+\infty$, which implies that $\|K(q)\|<+\infty$ and hence $\|S(q)\|<+\infty$. Thus $S(q)$ sends bounded sets into bounded sets. By a similar argument, we can show that the solutions of the equation $q=\lambda S(q)$, for $0<\lambda<1$, are bounded. Then by 
Schaefer's theorem the mapping has a fixed point.

\section{MAIN RESULT}

For $z \in F=C\left(J, \mathbb{R}^{n}\right)$, consider

$$
\begin{gathered}
x_{z}(t)=E(t, 0) x_{0}+P(t ; t) u_{0}+(1 / T)\left(u_{T}-u_{0}\right) \int_{0}^{t} P(s ; t) d s \\
+S(t ; T) Y_{z}(T)+\int_{0}^{t} E(t, s) g(s, z(s)) d s \\
u_{z}(t)=(1-t / T) u_{0}+(t / T) u_{T}+\bar{C}(t ; T) Y_{z}(T)
\end{gathered}
$$

where

$$
\begin{gathered}
Y_{z}(t)=[\bar{S}(T)]^{-1}\left[x_{T}-E(T, 0) x_{0}-P(T ; t) u_{0}-(1 / T)\left(u_{T}-u_{0}\right)\right. \\
\left.\quad \times \int_{0}^{T} P(s ; t) d s-\int_{0}^{T} E(T, s) g(s, z(s)) d s\right] .
\end{gathered}
$$

By a similar argument parallel to that of [1], we have the following propositions.

Proposition 2:

$$
\int_{0}^{t} E(t, s) B(s) u_{z}(s) d s=P(t ; t) u_{0}+(1 / T)\left(u_{T}-u_{0}\right) \int_{0}^{t} P(s ; t) d s+S(t ; T) Y_{z}(T)
$$

and $S(T ; T)=\bar{S}(T)$.

Proposition 3: $\quad$ Consider the boundary value control process

$$
\dot{x}(t)=A(t) x(t)+\int_{0}^{t} H(t, s) x(s) d s+B(t) u(t)+g(t, z)
$$

with

$$
\begin{array}{ll}
x(0)=x_{0}, & x(T)=x_{T} \\
u(0)=u_{0}, & u(T)=u_{T} .
\end{array}
$$

Then, if the matrix $M(0, T)$ is nonsingular, every pair $\left(x_{z}(t), u_{z}(t)\right)$ defined in (4) and (5) provides a solution of boundary value control process (6).

Now we shall prove the main result on the controllability of nonlinear Volterra integrodifferential system. 
Theorem 2: Assume that the nonlinear control system (1) satisfies the hypotheses and that the matrix $M(0, T)$ is nonsingular for $T>0$. Then, for every $\alpha, \beta$, $\gamma \in \mathbb{R}^{m}, x_{0}, x_{1}, x_{T} \in \mathbb{R}^{n}$, and every $\omega \in[0, T]$, there exists a control $v$, such that

(a) $\quad v(0)=\alpha, v(\omega)=\beta, v(T)=\gamma$

(b) the response of $(1)$, for which $x(0 ; v)=x_{0}$, satisfies $x(\omega ; v)=x_{1}, x(T ; v)=x_{T}$.

Proof: Consider the mapping $q: z \in F \rightarrow q(z)=x_{z} \in F$ where $x_{z}=x_{z}(t)$ and $x_{z}(t)$ and $u_{z}(t)$ are defined in (4) and (5) respectively. Then the proof is based upon two applications of Proposition 3.

First setting $\omega=T, u_{0}=\alpha, u_{T}=\beta, x_{T}=x_{1}$ we can obtain a response $x(t ; v)$ of (1) such that $x(0 ; v)=x_{0}$ and $x(\omega, v)=x_{1}$.

Then, setting $u_{0}=\beta, x_{0}=x_{1}, u_{T}=\gamma$ we can obtain a response $x(t ; v)$ of $(1)$ such that $x(\omega ; v)=x_{1}$ and $x(T ; v)=x_{T}$.

Thus we extend the response $x(t ; v)$ to whole interval $[0, T]$ and hence the theorem is proved.

To show that the mapping $q$ has a fixed point, we use Schaefer's theorem.

Since $E(\cdot, \cdot), P(\cdot ; \cdot)$ and $\bar{S}(\cdot)$ are continuous and $g(\cdot, z)$ is continuous with respect to $z$ we can say that $z \rightarrow Y_{z}(t)$ is continuous function with respect to $z$. Thus the map $z \rightarrow x_{z}$ is continuous. Assume that $\|z\| \leq r, 0<r<+\infty$. Then,

$$
\begin{gathered}
\|E(t, s)\| \leq \exp \left(a \omega+c \omega^{2} / 2\right) \\
\|P(t ; t)\| \leq b \omega \exp \left(a \omega+c \omega^{2} / 2\right) \\
\|\bar{C}(t ; T)\| \leq 2 b \omega^{2} \exp \left(a \omega+c \omega^{2} / 2\right) \\
\|S(t ; T)\| \leq 2 b^{2} \omega^{3} \exp \left(a \omega+c \omega^{2} / 2\right) \equiv a_{1} \\
\left\|Y_{z}(\omega)\right\| \leq a_{1}\left[\left\|x_{1}\right\|+\exp \left(a \omega+c \omega^{2} / 2\right)\left\|x_{0}\right\|\right. \\
+b \omega \exp \left(a \omega+c \omega^{2} / 2\right) \alpha \\
+|\alpha-\beta| b \omega \exp \left(a \omega+c \omega^{2} / 2\right) \\
\left.+\omega \exp \left(a \omega+c \omega^{2} / 2\right) \gamma\right] \\
\equiv a_{2} .
\end{gathered}
$$


Thus, we have

$$
\begin{gathered}
\|q(z)\|=\left\|x_{z}(t)\right\| \leq\left\|x_{0}\right\| \exp \left(a \omega+c \omega^{2} / 2\right)+\alpha b \omega \exp \left(a \omega+c \omega^{2} / 2\right) \\
+|\alpha-\beta| b \omega \exp \left(a \omega+c \omega^{2} / 2\right)+a_{1} a_{2} \\
+\omega \exp \left(a \omega+c \omega^{2} / 2\right) \gamma \equiv a_{3} .
\end{gathered}
$$

Let us now estimate,

$$
\begin{gathered}
\left\|x_{z}\left(t_{1}\right)-x_{z}\left(t_{2}\right)\right\| \leq\left\|E\left(t_{1}, 0\right)-E\left(t_{2}, 0\right)\right\|\left\|x_{0}\right\| \\
+\left\|P\left(t_{1} ; t_{1}\right)-P\left(t_{2} ; t_{2}\right)\right\||\alpha| \\
+\frac{|\alpha-\beta|}{T}\left\|\int_{t_{2}}^{t_{1}}\left(P\left(s ; t_{1}\right)-P\left(s ; t_{2}\right)\right) d s\right\| \\
+\left\|Y_{z}(\omega)\right\|\left\|S\left(t_{1} ; \omega\right)-S\left(t_{2} ; \omega\right)\right\| \\
+\left\|\int_{t_{2}}^{t_{1}}\left(E\left(t_{1}, s\right)-E\left(t_{2}, s\right)\right) g(s, z(s)) d s\right\| .
\end{gathered}
$$

From the previous inequalities, we have

$$
\begin{gathered}
\left\|E\left(t_{1}, 0\right)-E\left(t_{2}, 0\right)\right\| \leq\left|t_{1}-t_{2}\right|(a+c \omega) \exp \left(a \omega+\frac{c \omega^{2}}{2}\right) \\
\left\|P\left(t_{1} ; t_{1}\right)-P\left(t_{2} ; t_{2}\right)\right\| \leq\left|t_{1}-t_{2}\right| b \exp \left(a \omega+\frac{c \omega^{2}}{2}\right) \\
\left\|\int_{t_{2}}^{t_{1}}\left(P\left(s ; t_{1}\right)-P\left(s ; t_{2}\right)\right) d s\right\| \leq\left|t_{1}-t_{2}\right|^{2} b \exp \left(a \omega+\frac{c \omega^{2}}{2}\right) \\
\left\|S\left(t_{1} ; \omega\right)-S\left(t_{2} ; \omega\right)\right\| \leq\left|t_{1}-t_{2}\right|^{2} 2(a+c \omega) b^{2} \omega^{2} \exp 2\left(a \omega+\frac{c \omega^{2}}{2}\right) \\
\left\|\int_{t_{2}}^{t_{1}}\left(E\left(t_{1}, s\right)-E\left(t_{2}, s\right)\right) g(s, z(s)) d s\right\| \\
\leq\left|t_{1}-t_{2}\right|^{2} \gamma(a+c \omega) \exp \left(a \omega+\frac{c \omega^{2}}{2}\right) .
\end{gathered}
$$

Therefore,

$$
\begin{gathered}
\left\|x_{z}\left(t_{1}\right)-x_{z}\left(t_{2}\right)\right\| \leq\left\|x_{0}\right\|\left|t_{1}-t_{2}\right|(a+c \omega) \exp \left(a \omega+\frac{c \omega^{2}}{2}\right) \\
+|\alpha|\left|t_{1}-t_{2}\right| b \exp \left(a \omega+\frac{c \omega^{2}}{2}\right) \\
+\frac{|\alpha-\beta|}{\omega}\left|t_{1}-t_{2}\right|^{2} b \exp \left(a \omega+\frac{c \omega^{2}}{2}\right) \\
+\left|t_{1}-t_{2}\right|^{2}(a+c \omega) \gamma \exp \left(a \omega+\frac{c \omega^{2}}{2}\right)
\end{gathered}
$$




$$
\left.+a_{2} \mid t_{1}-t_{2}\right)^{2} 2 b^{2} \omega^{2}(a+c \omega) \exp 2\left(a \omega+\frac{c \omega^{2}}{2}\right) .
$$

Thus $z \rightarrow q(z)$ is an equibounded and equicontinuous mapping. Since the solutions of the equation $z=\lambda q(z)$ are bounded for $0<\lambda<1$, then by Schaefer's theorem, $q$ has a fixed point.

\section{REFERENCES}

[1] G. Anichini, "Global controllability of nonlinear control processes with prescribed controls", J. Optim. Theory Appl. 32, (1980), 2, pp. 183-199.

[2] G. Anichini, "Controllability and controllability with prescribed controls", J. Optim. Theory Appl. 39, (1983), 1, pp. 34-45.

[3] K. Balachandran, "Controllability of nonlinear Volterra integrodifferential systems", Kybernetika 25, (1989), 6, pp. 505-508.

[4] K. Balachandran and J.P. Dauer, "Controllability of nonlinear systems via fixed point theorems", J. Optim. Theory Appl. 53, (1987), 3, pp. 345-352.

[5] E.N. Chukwu and S.D. Silliman, "Complete controllability to a closed target set", J. Optim. Theory Appl. 21, (1977), 3, pp. 369-383.

[6] J.P. Dauer, "A controllability technique for nonlinear systems", J. Math. Anal. Appl. 37, (1972), 2, pp. 442-451.

[7] J.P. Dauer, "Controllability of nonlinear systems with restrained controls", J. Optim. Theory Appl. 14, (1974), 3, pp. 251-262.

[8] A.G. Kartsatos, "Global controllability of perturbed quasi-linear systems", Problems of Control and Information Theory 3, (1974), 2, pp. 137-145.

[9] D.L. Lukes, "Global controllability of nonlinear systems", SIAM Journal on Control 10, (1972), 1, pp. 112-126, Erratum, 11 (1973), p. 186. 


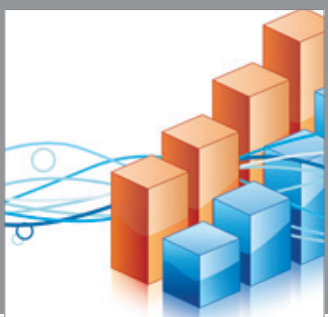

Advances in

Operations Research

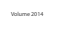

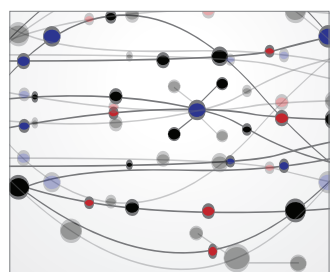

\section{The Scientific} World Journal
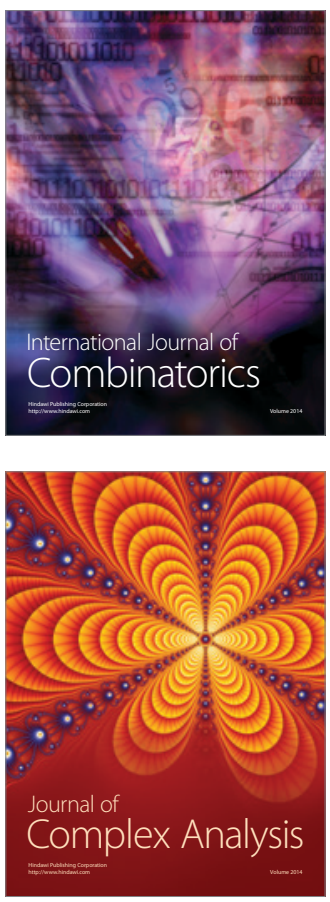

International Journal of

Mathematics and

Mathematical

Sciences
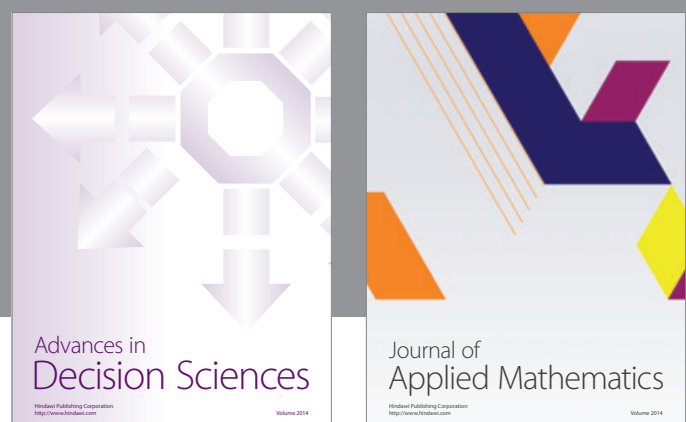

Journal of

Applied Mathematics
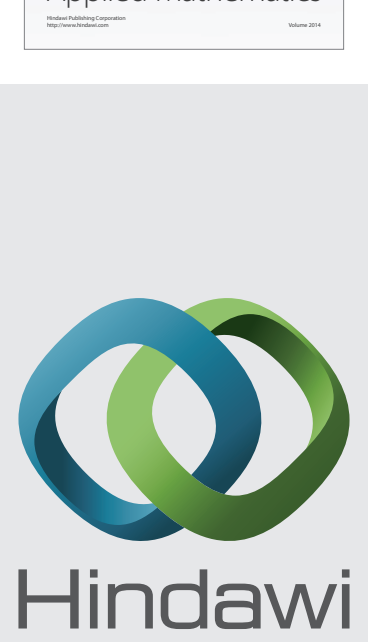

Submit your manuscripts at http://www.hindawi.com
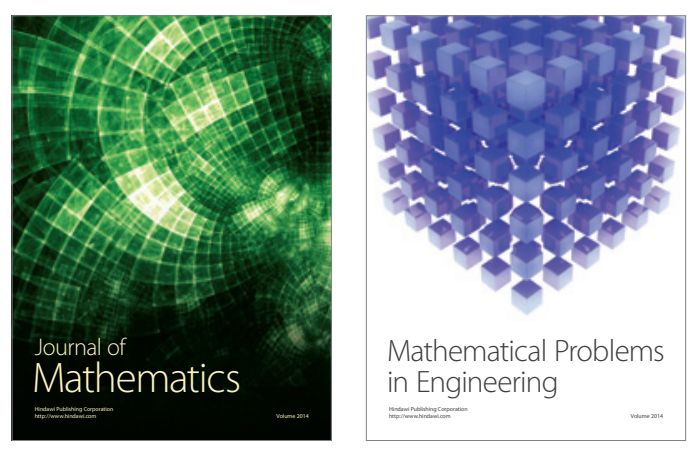

Mathematical Problems in Engineering
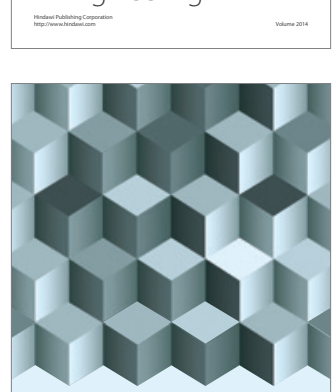

Journal of

Function Spaces
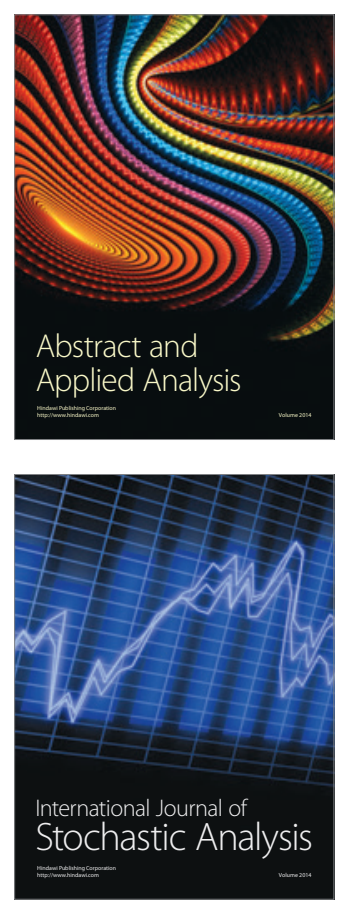

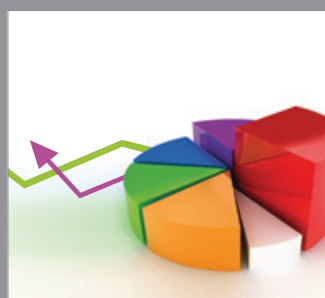

ournal of

Probability and Statistics

Promensencen
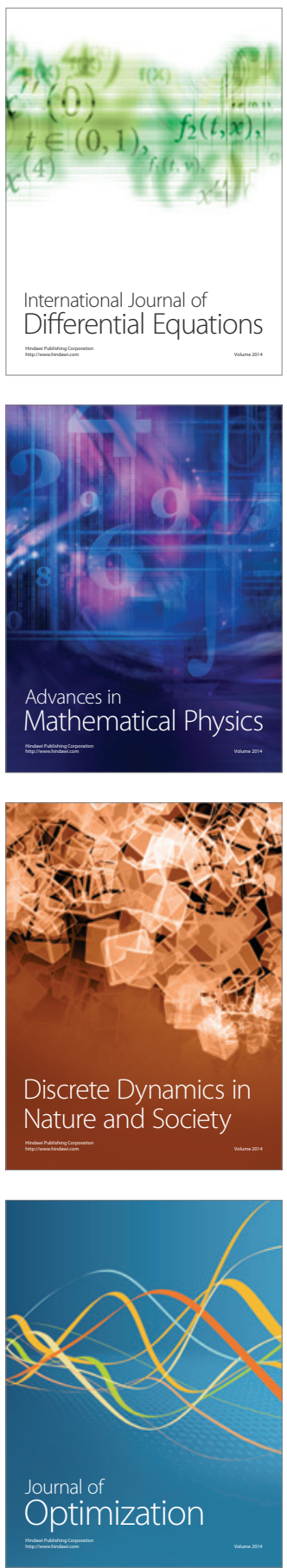